\title{
Fault Detection Methods in Air-Conditioning System Based on Parametric Statistics Characteristics
}

\author{
Yuan Liu',a, Pengcheng Zhao ${ }^{2, b}$, Wei Li ${ }^{3, c}$, Xiaozuo Lu ${ }^{1, d}$, Yimin Wang ${ }^{1, e *}$ \\ ${ }^{1}$ Tianjin University of Traditional Chinese Medicine, School of Chinese Medicine Engineering,88 \\ Yuquan Road, Tianjin, China
}

${ }^{2}$ Tianjin Architecture Design Institude, No. 95 Qixiangtai Road, Hexi District ,Tianjin, China

${ }^{3}$ Tianjin University of Traditional Chinese Medicine, College of Pharmaceutical Engineering for Chinese Medicine, College of Traditional Chinese Medicine, 88 Yuquan Road, Tianjin, China

ales314@sina.com, bzhaopccd@126.com, ‘67846874@qq.com, d13702093979@163.com, e*ylzmyh@163.com

Keywords: Air-conditioning systems; hybrid system model; fault detection; ROC analysis

Abstract: Fault detection for air-conditioning system was the key to guarantee performance and energy-saving. However, it was hard to resolve the parameter drift, multiple modes, incipient failures and existing failures via the conventional fault detection for air-conditioning system. This paper aimed at air-conditioning system with identical units and established hybrid mathematical models. The characteristic parameters of similar units was obtained by the Interactive Multi-mode(IMM) filter further processing parameter statistical properties of similar units, according to statistical properties of the parameters detect fault in the system. The results demonstrated that the fault detection method can be used to effectively detect incipient failures and preexisting failures in the air-conditioning system under the conditions of parameter drifting.

\section{Introduction}

Fault diagnosis of air conditioning units affect the control performance of the system and energy saving.

At present, the main methods of fault detection of air conditioning units include methods based on analytic model ${ }^{[2]}$, knowledge-based reasoning methods ${ }^{[3]}$ and data-driven methods ${ }^{[5,6]}$. Methods based on the analytic model require the establishment of accurate mathematical model, comparing the actual system and the corresponding model to obtain the residual error and detecting failure by the residual error. For example, [2] modeled the central air conditioning system from the mass-energy relationship, and carried out fault detection. Methods of knowledge-based reasoning requires sophisticated expertise to build a complete set of rules, whose reliability depends on the establishment of relationships or rules. For example [3] establish the fault detection rules based on the relationship between the temperature and humidity of different location of the air conditioning unit. The data-driven fault detection method does not need to establish accurate mathematical model or rule base, it mining and analysis the hidden information to detect system fault or sensor fault through the processing data of the system. In recent years, the data-driven approach has become a hot research area $^{[7]}$, which is widely used in fault detection of air-conditioning system because of the convenient data acquisition and difficulty in establishing accurate mathematical models or rules. For example reference [5] used Principal component analysis (PCA) to detect sensor fault of Constant air volume air conditioning (CAV). The neural network method was widely applied to diagnosis sensor fault of air-conditioning system ${ }^{[6]}$, Data-driven methods are also widely used in detecting other types of fault [8-11].

The main difficulties of Air conditioning unit fault detection are parameter drift, multi-mode, gradual failure and existing faults. Li studied the problem of identification of multiple unknown modes under parametric drift in [1]. This paper studies the fault diagnosis scheme of the air 
conditioning unit based on the principle of the same parameter statistic characteristics of the same mode, mainly studies the gradual fault and the existing fault detection.

There are a large number of air conditioning units with similar operating environments in the large public buildings such as office building, library and so on. Most of the existing fault detection methods only detect faults from the data and characteristics of the unit itself, which result in poor detection of the gradual faults and existing faults. And different units have different operation mode and working state, making direct use measurement data of other units impossible. same model unit is extracted by the method of reference [1], and the external data of multiple similar units are mapped to the common parameter space, and the fault detection of each unit is detected by establishing the common distribution of similar units.

\section{Modeling}

The air-conditioning unit usually includes a finite number or countable modes, and these modes are discrete, the conversion is random, the conversion between these modes has no after-effects, which meet the characteristics of the Markov jump system. Therefore, the operation mode of air-conditioning unit can be described by a Markov chain. A large number of similar air-conditioning units are included in the large buildings, these similar units have some common parameters which subject to an identical distribution. Because of the commonness and difference of each unit, the system variables can be divided into two parts: the global state variable and the local state variable, which are described by two state equations respectively. Taking the existence of interference into consideration, we introduce independent Gaussian white noise which represent the process noise and measurement noise respectively. Each subsystem can be described by hybrid system model as follows ${ }^{[13]}$ :

$$
\begin{aligned}
& \xi_{k+1}^{(s)}=f_{k}^{(s)}\left(\xi_{k}^{(s)}, \theta_{k}^{(s)}, m_{k}^{(s)}, \omega_{\zeta_{k}}^{(s)}\right) . \\
& \theta_{k+1}^{(s)}=g_{k}\left(\theta_{k}^{(s)}, m_{k}^{(s)}, \omega_{\theta_{k}}^{(s)}\right) . \\
& z_{k+1}^{(s)}=h_{k}^{(s)}\left(\xi_{k}^{(s)}, \theta_{k}^{(s)}, v_{k}^{(s)}\right) .
\end{aligned}
$$

Where $k=0,1,2, \cdots$ is the time index, $s=0,1,2, \cdots S$ is the unit index, $x_{k}^{(s)}$ is the base-state vector of unit $\mathrm{s}$, and $w_{k}^{(s)} \square N\left(0, Q_{k}^{(s)}\right) \quad v_{k}^{(s)} \square N\left(0, R_{k}^{(s)}\right)$ represent independent Gaussian process noise and measurement noise. And $g_{k}$ is the common state transfer function of all units with similar characteristics ${ }^{[14]}$.

The operation mode of unit $\mathrm{s} m_{k}^{(s)} \in\{1,2 \cdots M\}$ is modeled by a Markov chain with state $\mathrm{s}$ and initial transition probabilities are as follows, respectively

$$
\begin{aligned}
& P\left\{m_{0}^{(s)}=i\right\}=\pi_{0} . \\
& P\left\{m_{k+1}^{(s)}=j \mid m_{k}^{(s)}=i\right\}=\pi_{i j} .
\end{aligned}
$$

For $i, j=1,2, \cdots M$, and $\pi_{i j}$ meet $\sum_{j=1}^{N} \pi_{i j}=1, i=1,2, \cdots, M$.

The three-way valve of air conditioning unit have three working modes: Cooling mode, Ventilation mode and Heating mode, corresponding to $m=1,2$ and 3 . The parameters of the model have the same distribution when the units work in the same mode. In this paper, $a_{i}$ and $b_{i}$ are defined as autoregressive parameters and moving average parameters, then we have a sliding autoregressive model: 


$$
z_{k}=a_{1} z_{k-1}+a_{2} z_{k-2}+b_{1} s_{k-1}+b_{2} s_{k-2}
$$

Where $s_{k-1}$ is valve position of time $k-1, z_{k}$ is return air temperature of time $k$.

Make $x_{k}=\left[\begin{array}{lll}a_{1} & a_{2} & \mathrm{~b}_{1} \\ \mathrm{~b}_{2}\end{array}\right]_{k}^{T} A_{k}=I \quad C_{k}=\left[z_{k-1} z_{k-2} s_{k-1} \mathrm{~s}_{k-2}\right]$, Then the above-mentioned difference equation can be rewriten as' a state-space equation as shown in eq.7:

$$
\begin{aligned}
& x_{k}=A_{k} x_{k-1}+Q^{\frac{1}{2}} \omega_{k} \\
& z_{k}=C_{k} x_{k}+R^{\frac{1}{2}} v_{k} .
\end{aligned}
$$

Where $\omega_{k}, v_{k} \sim N\left(0,1^{2}\right)$ are noises, $\mathrm{Q}$ and $\mathrm{R}$ which need to be seleced appropriately are the system noise covariance matrix and the measurement noise covariance matrix respectively.

For $m=1$ and $m=3$ (Cooling mode and Heating mode)

$$
\theta_{k}^{(m, s)} \square\left[a_{1} a_{2} \mathrm{~b}_{1} \mathrm{~b}_{2}\right]_{k}^{T(m, s)} .
$$

For $m=2$ (Ventilation mode), we can directly construct the parameter vector as:

$$
\theta_{2}^{(m, s)} \square\left[\begin{array}{llll}
1 & 0 & 0 & 0
\end{array}\right]_{2}^{T} \text {. }
$$

There is no local state variable in all modes in this case,i.e.: $\varepsilon_{k}^{(m, s)}=$ [empty].

The ultimate goal of fault detection is detection if there is at least one unit has changed among units $\mathrm{S}, \mathrm{S}=1,2, \ldots, \mathrm{M}$ based on the observation data $Z_{k}=\left\{z_{k}^{(s)}: s=1,2, \ldots, N\right\}_{k=0}^{k}$, After creating the air conditioning unit model, detect system fault as the following steps:

1) Obtain the statistical properties of parameters $\theta_{0}^{(s)}$ and $\omega_{\theta_{k}}^{(s)}$ in all the units by IMM filtering and construct the multiple Gaussian distributions of the similar parameters $\theta_{k}$.

2) Calculate the test statistics of each unit, according to confidence coefficient and statistical distribution to determine the test threshold, and then judge each unit is failure or not by residual chi-square test results.

\section{IMM filtering}

At present, multiple model filtering (MM) is the main method of state estimation for hybrid systems. The system model established in Section 2 takes the air conditioning unit model parameters $x_{k}$ as the state variables, but $x_{k}$ cannot be directly measured, we need to filter the measurement data to obtain the base-state vector $x_{k}$. Air-conditioning units has multiple working modes and there are interaction between the modes, so we choose the interactive multi-mode filtering method (IMM) to filter ${ }^{[15]}$.

The basic idea of the IMM algorithm is that at each moment, mix the state estimate values of all filters at the previous time to obtain the initial conditions which are matched with each model, and then filter each model synchronous. Finally, update the model probabilities based on model matching likelihood function, and combine the revised state estimation value (weighted sum) of all the filters as output. IMM algorithm is a recursive algorithm, each cycle generally includes four steps: interactive computing, model conditional filtering, model probability update and combine estimate. The detailed procedures of the algorithm are shown in Fig1.

The base-state vector of every unit $\mathrm{x}_{k}^{(s)}=\left[\xi_{k}^{(s) T}, \theta_{k}^{(s) T}\right]^{T}$ can be obtained by the commonly used IMM filtering algorithm. The common parameters of similar units follow an identical distribution.

Take the interference into consideration, for a single model, $\theta_{\mathrm{k}}^{(s)}$ should be subject to multiple normal distribution. But for the hybrid system (including multiple models), the distribution of $\theta_{\mathrm{k}}^{(s)}$ cannot be expressed by a single Gaussian distribution, and Mixed Gaussian distribution should be used to express the distribution. 
Take the unit working in the cooling state as an example, We collected 21 groups of temperature and valve opening data in an experiment as shown in Table 1.

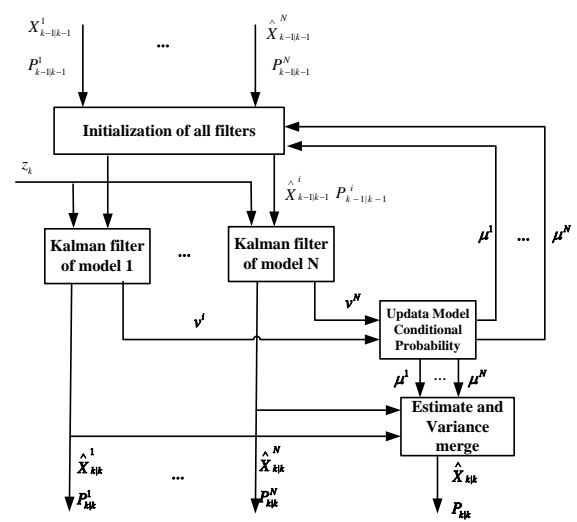

Fig.1 Step of IMM filter

Table 1 Temperature and valve position data under cooling mode

\begin{tabular}{llllll}
\hline $\mathrm{K}$ & $\mathrm{Z}(\mathrm{K})$ & $\mathrm{Z}(\mathrm{K}-1)$ & $\mathrm{Z}(\mathrm{K}-2)$ & $\mathrm{S}(\mathrm{K}-1)$ & $\mathrm{S}(\mathrm{K}-2)$ \\
0 & 49.46 & 49.46 & 49.46 & 0 & 0 \\
1 & 44.86 & 49.46 & 49.46 & -10.00 & 0 \\
2 & 42.70 & 44.86 & 49.46 & -10.00 & -10.00 \\
3 & 41.08 & 42.70 & 44.86 & -10.00 & -10.00 \\
4 & 40.27 & 41.08 & 42.70 & -10.00 & -10.00 \\
5 & 39.46 & 40.27 & 41.08 & -10.00 & -10.00 \\
6 & 38.65 & 39.46 & 40.27 & -10.00 & -10.00 \\
7 & 38.11 & 38.65 & 39.46 & -10.00 & -10.00 \\
8 & 37.57 & 38.11 & 38.65 & -10.00 & -10.00 \\
9 & 37.03 & 37.57 & 38.11 & -10.00 & -10.00 \\
10 & 36.49 & 37.03 & 37.57 & -10.00 & -10.00 \\
11 & 35.95 & 36.49 & 37.03 & -10.00 & -10.00 \\
12 & 35.41 & 35.95 & 36.49 & -10.00 & -10.00 \\
13 & 35.14 & 35.41 & 35.95 & -10.00 & -10.00 \\
14 & 34.59 & 35.14 & 35.41 & -10.00 & -10.00 \\
15 & 34.32 & 34.59 & 35.14 & -10.00 & -10.00 \\
16 & 33.78 & 34.32 & 34.59 & -10.00 & -10.00 \\
17 & 33.51 & 33.78 & 34.32 & -10.00 & -10.00 \\
18 & 33.24 & 33.51 & 33.78 & -10.00 & -10.00 \\
19 & 32.70 & 33.24 & 33.51 & -10.00 & -10.00 \\
20 & 32.43 & 32.70 & 33.24 & -10.00 & -10.00 \\
\hline
\end{tabular}

The global state variables $x_{k}=\left[\begin{array}{lll}a_{1} & a_{2} & \mathrm{~b}_{1} \\ \mathrm{~b}_{2}\end{array}\right]_{k}^{T}$ obtained by IMM filtering are shown in Fig. 2 .

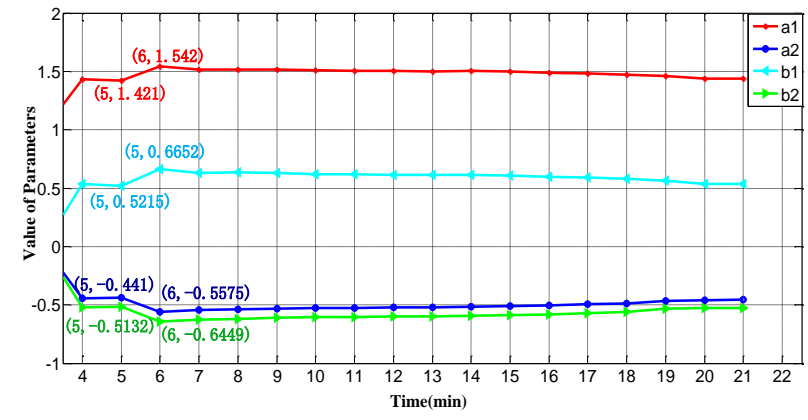

Fig. 2 The result of IMM filter under cooling mode 


\section{Fault detection}

Units that operate in the same mode can be found by clustering algorithms ${ }^{[1]}$, and then the common distribution of the base-state vector $x_{k}$ can be established by the filtering results of the similar units. The established statistical model is used as the model of the fault-free hypothesis $H_{0}$ to detect fault or not of each unit.

Considering the fact that the model of fault hypothesis $\mathrm{H}_{1}$ is absent in fault detection process of air conditioning unit, generalized likelihood ratio method cannot be used to detect the failure of air conditioning unit, and the residual chi-square test is adopted to detect fault of air conditioning. Assuming that the measurement sequence is $z_{k}$, the sequence of the filtered values is $\hat{z}_{k \mid k-1}$, and the measurement residual sequence is $\widetilde{z}_{k}$, where $\tilde{z}_{k}=z_{k}-\hat{z}_{k \mid k-1}$. As we know that $\tilde{z}_{k} \sim N\left(0, S_{k}\right)$, where $S_{k}=\operatorname{cov}\left(\tilde{z}_{k}\right)$. make

$$
\delta_{k}=\tilde{z}_{k}^{\prime} S_{k}^{-1} \tilde{z}_{k}
$$

Then $\delta_{k}$ Subject to a chi-square distribution with $n_{z}$ degrees of freedom, i.e. $\delta_{k} \sim \chi_{n_{z}}^{2}$, where $n_{z}=\operatorname{dim}\left(\tilde{z}_{k}\right)$.Perform a hypothesis test on the statistics $\delta_{k}$, when $\delta_{k}>\chi_{n_{z}}^{2}(\alpha)$, hypothesis $H_{0}$ will be rejected by the reliability $1-\alpha$, namely the system has failed.

In the process of fault detection, the parameters $x_{k}$ are performance parameters of three-way valve, cannot be measured directly. The common distribution parameters of the units work in the same mode are treated as real value, and the model parameters of the unit which is tested can treated as measured value. And the difference between the two parameters is regarded as a residual $\widetilde{\mathrm{z}}_{\mathrm{k}}$, normalize $\widetilde{\mathrm{z}}_{\mathrm{k}}$ to get $\delta_{k}$, and perform a chi-square test on $\delta_{k}$. Considering that the amount of computation increases significantly over time, the estimation of the time of failure occured is limited to a "sliding window" with a certain width. Commonly use $\delta_{k}^{s}$ (sum of $\delta_{k}$ in a sliding window with width s) and the exponential decay average value $\delta_{k}^{\rho}$ as a statistic to detect fault.

$$
\begin{aligned}
& \delta_{k}^{s}=\sum_{j=k-s+1}^{k} \delta_{k} . \\
& \delta_{k}^{\rho}=\sum_{j=1}^{k} \rho^{k-1} \delta_{j}=\rho \delta_{k-1}^{\rho}+\delta_{k} .
\end{aligned}
$$

Obviously, $\delta_{k}^{s}$ obeys the chi-square distribution $\left(\delta_{k}^{s} \sim \chi_{s n_{z}}^{2}\right)$. The decay parameter is $\rho=(s-1) / s$ where $s$ is the width of sliding window. Select an appropriate $\delta_{0}^{\rho}$ (to prevent considering reinitialization as fault), we can get $\delta_{k}^{\rho}$ through a recursive algorithm. Select the threshold $\gamma_{f}$, and the system is considered to be faulty when $\delta_{k}^{\rho}>\gamma_{f}$.

\section{Simulation}

\section{Simulation model}

It can be seen from the above that the fault detection of the air conditioning unit is finally carried out by analyzing the statistical characteristics of the air conditioning model parameters obtained by filtering. In this paper, the fault detection algorithm is simulated under the premise of the known parameter distribution characteristics.

In order to observe the performance of the algorithm, a simple model that conforms to a real system is constructed based on the conclusion in [1] that the parameters of the similar units obey the same distribution. These simple models have no local state $\xi$ (the local state $\xi$ does not affect the result of fault detection), and global variables of each unit have been obtained by IMM filtering. Taking the existence of error and interference into account, the parameters obtained after filtering obey the standard multivariate normal distribution, expressed as follows: 


$$
\theta_{k} \sim N\left(\left[\begin{array}{l}
A \\
B
\end{array}\right],\left[\begin{array}{ll}
1 & 0 \\
0 & 1
\end{array}\right]\right)
$$

Assuming the global parameters of a single model $\theta=[A, B]^{\prime}$, there is restricted difference among the parameters of each unit, without loss of generality, suppose $\theta$ obeys the following distribution ${ }^{[13]}$ :

$$
\theta^{(1)} \sim N\left(\left[\begin{array}{c}
-1 \\
1
\end{array}\right],\left[\begin{array}{cc}
0.25^{2} & 0 \\
0 & 0.25^{2}
\end{array}\right]\right)
$$

In the simulation, the following faults are introduced for a few units.

Gradual fault: At time $k_{F}=5$, unit running in Mode 1 enter to fault state:

$$
\theta_{k}^{(1,1)}=\theta_{k-1}^{(1,1)}+\left[\begin{array}{c}
0.2 \\
0
\end{array}\right], k \geq k_{F}
$$

Existing failures: Unit 1 running in Mode 1 has failed and the initial parameter vector is:

$$
\theta_{1}^{(1,1)} \sim N\left(\left[\begin{array}{l}
0 \\
1
\end{array}\right],\left[\begin{array}{cc}
0.25^{2} & 0 \\
0 & 0.25^{2}
\end{array}\right]\right)
$$

\section{Simulation results}

In this paper, receiver operating characteristic curve ${ }^{[19]}$ (ROC) is used to analyze the performance of the proposed fault detection algorithm. As the main study of this paper is gradual fault and the existing fault, respectively, the following simulation to verify the fault detection algorithm for pre-exisiting fault and the gradual fault detection.

\section{Gradual fault detection}

Use Matlab programming to generate a number of groups with 50 independent similar units, and introduce gradual fault as shown in Eq. 15. Select the fault detection window size $2(\rho=0.5)$. The frequency of true positive class(TP), false negative class $(\mathrm{FN})$, false positive type (FP), true negative class $(\mathrm{TN})$ are counted and the false positive rate $(\mathrm{FPR}=\mathrm{FP} /(\mathrm{TP}+\mathrm{FN}))$ and the true positive rate $(\mathrm{TPR}=\mathrm{TP} /(\mathrm{TP}+\mathrm{FN}))$ were calculated for different thresholds. Draw the ROC curve as shown in Fig. 3.

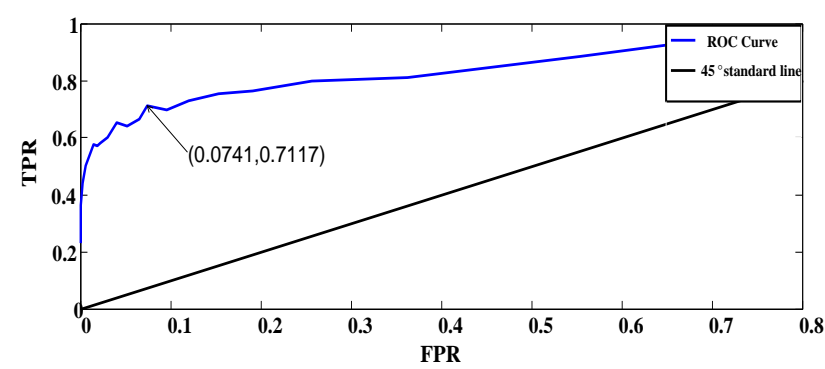

Fig. 3 ROC curve of gradual FD when units $=50, s=2(\rho=0.2)$

From Fig.3, it can be seen that the ROC curve is above the 45 degrees standard curve and away from the standard curve, indicating that the fault detection scheme is valid. At the same time, we can see that when (false positive rate, true positive rate) at (0.0741-0.7117), that is, the threshold at 0.04 the fault detection effect achieve the best.

Change the size of the fault detection window to $3(\rho=0.5)$ and $5(\rho=0.8)$, respectively. Changing the threshold value, calculate false positive rate and true positive rate under different threshold and draw ROC curve shown in Fig. 4. 


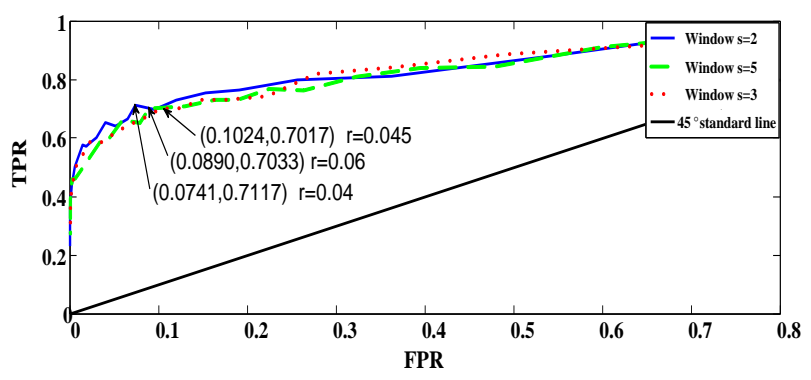

Fig. 4 ROC curve of gradual FD under variational window size

From Fig. 4, it can be seen that the three ROC curves above the standard curve of 45 degrees, and away from the standard curve, indicating the use of fault detection scheme effective. At the same time, changing the size of the detection window will affect the effect of fault detection. And the best threshold varies with the detection window, the two parameters should be matched to achieve the best detection results.

\section{Exisiting fault detection}

Use Matlab programming to generate a number of groups with 50 independent similar units, and introduce pre-exisiting fault as shown in Eq. 16. Select the fault detection window size $2(\rho=0.5)$. The frequency of true positive class(TP), false negative class (FN), false positive type (FP), true negative class (TN) are counted and the false positive rate and the true positive rate were calculated for different thresholds. Draw the ROC curve as shown in Fig. 5.

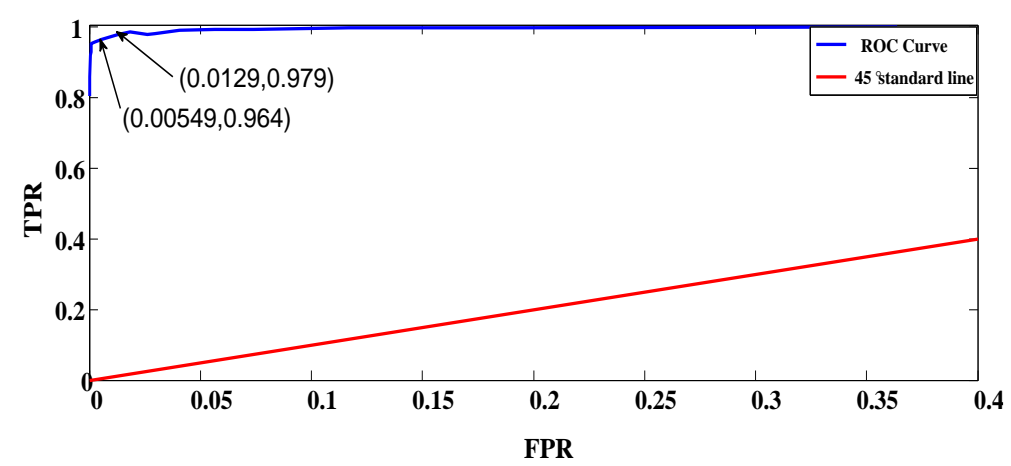

Fig.5 ROC curve of incipient FD when units $=50, \mathrm{~s}=2(\rho=0.2)$

From Fig. 5, it can be seen that the ROC curve is above the standard curve of 45 degrees and far away from the standard curve, which shows that the fault detection scheme is very effective. At the same time, we can see that when (false positive rate, true positive rate) at (0.0129-0.979), that is, the threshold at 0.02 the fault detection effect achieve the best.

\section{Conclusions}

The gradual faults and existing faults of air-conditioning units are always the difficulties of fault detection. The existing fault detection methods neglect the characteristics of large numbers of similar units in large public buildings. In this paper, the method of comparing the statistical characteristics of a large number of similar units' parameters for fault detection of air conditioning, effectively solve the existing fault and gradual fault detection. A new type of fault detection method based on the common distribution of similar units is proposed by introducing a special hybrid system mathematical model to describe the air conditioning system with a large number of similar units. This method is applicable not only to fault detection of air conditioning units, but also to other large systems with multiple similar units. Simulation results show that the proposed method can effectively detect both gradual faults and existing faults. 


\section{Acknowledgements}

This work was financially supported by the National Basic Research Program of China (973 Program) (2014CB542902), Undergraduate Teaching Quality \& Teaching Reform Research Program of Tianjin Ordinary Colleges and Universities (A07-1104) and National Key Technologies R\&D Program (2012BAI25B05).

\section{References}

[1] Donghui Li, Penglin He. Atca Electronica Sinica, 2014,10(42):2004-2008. In Chinese

[2] Vasso Reppa, Panayiotis Papadopoulos, Marios M. Polycarpou, Christos G. Panayiotou. IEEE Transactions on Control Systems Technology,2015,23(4):1323-1337.

[3] Yi Jiang, Weifeng Zhu. T singhua Univ ( Sci \& Tech),1999,39(12):54-56,61. In Chinese

[4] X R Li , V P Jilkov. IEEE Transactions on Aerospace and Electronic Systems, 2005, 41 (4):1255 $-1321$.

[5] Donghui Li, Leying Wang, Sheng Li. electronic technology, 2008,23 (6): 130-136. In Chinese

[6] Donghui Li, Hao Liu. Transactions of China Electrotechnical Society, 2005, 20(5):49-52. In Chinese

[7] Han Li, Deyun Xiao. Control and Decision, 2011,26(1):1-9. In Chinese

[8] Yuanzhang Wang, Chunhua Wu, Diqing Zhou, et.al. Power System Protection and Control, 2013,41(16):108-114. In Chinese

[9] Liping Shi, Jiasheng Tang, Panpan Wang,et.al. Transactions of China Electrotechnical Society ,2015,30(24):38-45. In Chinese

[10] Huiming Yang, Jiang Cui, Zhuorang Zhang,et,al. Transactions of China Electrotechnical Society, 2014 (S1): 164-169. In Chinese

[11] Jinliang Yin, Yongli Zhu, Guoqin Yu,et,al. Transactions of China Electrotechnical Society, 2013,28(1):158-164.

[12] Yu X, Liu Y, Wei D, et al. A hybrid markov model based on EM algorithm[C]//Control, Automation, Robotics and Vision, 2006. ICARCV'06. 9th International Conference on. IEEE, 2006: 1-5.

[13] Anwer Bashi, Vesselin P Jilkov , X Rong Li. IEEE Transactions on Control Systems Technology, 2011, 19(5):957-968.

[14] J Ru, X Rong Li. IEEE Transactions on Control Systems Technology,2008,16(5):1029-1038.

[15] Y Zhang, XR Li. IEEE Transactions on Aerospace and Electronic Systems, 1998, 32(4): 1293-1312.

[16] Anwer Bashi, Vesselin P Jilkov,X Rong Li. Fault Detection for Systems with Multiple Unknown Modes and Similar Units - Part I[C]. International Conference on Information Fusion. Seattle:WA,2009.732-739.

[17] Anwer Bashi, Vesselin P Jilkov, X Rong Li. Fault Detection for Systems with Multiple Unknown Modes and Similar Units - Part II: application to HVAC[C]. International Conference on Information Fusion, Seattle:WA,2009.740-747.

[18] Haosu Shi. Electronic Design Engineering, 2010,18 (9):36-39. In Chinese 